\title{
Labyrinthe
}

\section{Translations analogiques : la Rome impériale, l'Angleterre médiévale et les Indes britanniques}

\section{Ananya Jahanara Kabir}

Traducteur : Laurence Marie

\section{(2) OpenEdition}

\section{Journals}

Édition électronique

URL : http://journals.openedition.org/labyrinthe/4088

DOI : $10.4000 /$ labyrinthe.4088

ISSN : 1950-6031

Éditeur

Hermann

Édition imprimée

Date de publication : 28 août 2010

Pagination : 87-112

ISBN : 9782705669973

Référence électronique

Ananya Jahanara Kabir, «Translations analogiques : la Rome impériale, l'Angleterre médiévale et les Indes britanniques », Labyrinthe [En ligne], 35 | 2010 (2), mis en ligne le 27 juillet 2012, consulté le 30 avril 2019. URL : http://journals.openedition.org/labyrinthe/4088; DOI : 10.4000/labyrinthe.4088 


\title{
Translations analogiques : la Rome impériale, l'Angleterre médiévale et les Indes britanniques
}

\author{
Ananya Jahanara KABIR \\ Contact : a.j.kabir@leeds.ac.uk
}

\begin{abstract}
○ 2005 Cambridge University Press. La version originale de ce texte est parue dans Ananya Jahanara Kabir, Deanne Williams (dir.), Postcolonial approaches to the European Middle Ages: translating cultures, Cambridge, Cambridge University Press, 2005, p. 183-203. Reproduction sur autorisation.
\end{abstract}

Caractacus, Cassibelauneas et Boadicée représentent le stade primitif du sentiment natif indien. Les « gémissements des Bretons » suppliant les Romains de continuer à les protéger représentent la nouvelle ère qui se lève sur l'Inde.

Sir Charles Trevelyan, 1843-1844

\begin{abstract}
À première vue, il ne fait aucun doute que cette analogie évocatrice, qui associe la Bretagne dominée par Rome et les Indes britanniques, et telle que l'emploie l'administrateur colonial, écrivain et homme politique Charles Trevelyan lors d'une discussion parlementaire portant sur les bénéfices qu'il y aurait à enseigner l'anglais aux Indiens, reprend l'un des tropes les plus récurrents de l'Empire britannique : l'idée que celui-ci se façonne à l'image de la Rome impériale ${ }^{1}$. Les traces matérielles de ce trope restent clairement visibles dans l'espace post-impérial aussi bien que postcolonial.

« Le temps semble être venu de dire le monde français, comme autrefois le monde romain ; et la Philosophie, lasse de voir les hommes toujours divisés par les intérêts divers de la politique, se réjouit maintenant de les voir, d'un bout de la terre à l'autre, se former en république sous la domination d'une même langue. Spectacle digne d'elle, que cet uniforme et paisible empire des lettres qui s'étend sur la variété des peuples, et qui, plus durable et plus fort que l'empire des armes, s'accroît également des fruits de la paix et des ravages de la guerre !» Antoine Rivarol, De l'Universalité de la langue française, Berlin / Paris, Bailly, 1784, p. 1-2.
\end{abstract}


La statue érigée dans la cathédrale Saint-Paul à Londres, qui montre le pionnier des orientalistes William Jones en toge et appuyé sur les deux volumes des Codes de Menu, trouve un écho manifeste dans les jardins du Mémorial Victoria de Calcutta, où se dresse encore aujourd' hui un contemporain de Jones, le gouverneur général pro-orientaliste Warren Hastings, vêtu en sénateur romain et flanqué d'un brahmine pandit et d'un musulman maulvi, plongés respectivement dans l'étude d'un manuscrit sanscrit et d'un manuscrit perse ${ }^{2}$. Juxtaposés à ces rappels métonymiques de leur conquête linguistique de l'Inde, les figures romanisées de Jones et de Hastings concentrent puissamment - et communiquent - les relations idéologiques qui unissent « la gouvernance de la langue et la langue de la gouvernance », la pax Romana et la pax Britannica, les formes romaine et britannique de la translatio studii et imperii.

De manière évidente, ces relations sont à l'arrière-plan de l'analogie de Trevelyan, qui est elle-même une forme de translation culturelle. Il n'en reste pas moins que, derrière cette concurrence confiante entre l'impérialisme linguistique et l'impérialisme culturel, s'expriment des angoisses plus profondes qui ont partie liée avec la généalogie et les origines, et qui, de manière symptomatique, interrompent le processus de l'analogie comme translation, ainsi que le suggère la troublante référence aux «gémissements des Bretons ». Traduisant l'expression «gemitus Britannorum » qui, selon Gildas, historien insulaire du $v^{\mathrm{e}}$ siècle, fait référence à la façon dont les Bretons se caractérisent eux-mêmes dans la supplique qu'ils adressèrent au consul romain Aetius $^{3}$ et qui resta sans réponse, les « gémissements des Bretons » évoqués par Trevelyan viennent interrompre et mettre à mal l'équation familière qui unit les Indes britanniques et la Rome impériale ${ }^{4}$. En nous invitant à relire cette équation, ils donnent à voir ce que Homi Bhabha a appelé « cet oubli — la signification d'un moins dans l'origine — qui constitue le début du récit de la nation ${ }^{5} »$. N'en déplaise à Bhabha et consorts, les médiévistes ont démontré d'une manière de plus en plus convaincante que le principal récit de la Nation [européenne] dépendait d'un processus non seulement d'estrangement de ses colonies, mais aussi d' «oubli » de son passé médiéval, en vue de mettre sa modernité entre parenthèses ${ }^{6}$. J'entends souligner que ce «processus d'oubli » a donné lieu à des manœuvres bien plus complexes qu'une simple disjonction entre le médiéval et le moderne - notamment en raison de l'analogie concomitante qui asso- 


\section{Translations analogiques}

ciait la Bretagne et Rome, et aussi de la perception d'une relation entre Rome et l'Europe médiévale.

\begin{abstract}
« C'est entendu, il y a des connexions partout - mais pourquoi alors persistons-nous à réduire des phénomènes dynamiques, interconnectés, à des choses immobiles et sans liens entre elles ? Quelque chose là dedans tient peut-être à la manière dont nous avons appris notre propre histoire. Que nous enseigne-t-on, en classe et au dehors ? Qu'il existe une entité appelé l'Occident ; qu'il s'agit d'une société et d'une civilisation pouvant être conçues indépendamment des autres, et en opposition à elles. Nombreux parmi nous sont ceux qui ont grandi dans l'idée que la généalogie de cet Occident pouvait être reconstituée ainsi : la Grèce engendra Rome, Rome l'Europe chrétienne, l'Europe chrétienne la Renaissance, la Renaissance les Lumières, les Lumières la démocratie politique et la révolution industrielle. Croisée avec la démocratie, l'industrie produisit à son tour les États-Unis, incarnation des droits à la vie, à la liberté et à la poursuite du bonheur ».

Eric R. Wolf, Europe and the People without history, Berkeley, University of California Press, 1982, p. 4-5.
\end{abstract}

en créant par la même occasion un système de coordonnées spatiotemporelles pour le moi impérial d'après les Lumières. Comment les empires britannique et romain se sont-ils insérés dans cette grille de lecture ? Est-elle entrée en concurrence avec le médiévalisme impérial ou s'est-elle associée avec lui ?
Comme je le montre ailleurs de manière détaillée, le Moyen Âge a offert aux Britanniques installés en Inde un outil pour dépasser le choc ontologique provoqué par la confrontation coloniale ${ }^{7}$. Construire le présent étranger à l'image d'un passé délibérément « oublié » a de fait permis de faire cohabiter le présent avec le passé,

" [Au cours du premier $x \mid x^{e}$ siècle] la vie universitaire britannique, si tant est qu'il en existât vraiment une, était dominée par les études bibliques et classiques. Les secondes, en particulier, prospéraient. Depuis la fin du XvIII siècle, la culture grecque et romaine avait connu un regain d'intérêt, nourri en partie par l'enthousiasme des Romantiques envers la révolte grecque contre les Turcs, par leur culte des ruines et la découverte, au milieu du siècle, des vestiges de Pompéi, et, surtout, par le rôle croissant des public schools (autrement dit des établissements privés réservés aux plus riches), au sein desquelles nul doute n'était permis : pour se forger le caractère, il fallait étudier les classiques. [...] Le grec et le latin devaient former l'esprit et la conscience citoyenne. Pour ceux qui furent appelés à diriger le Raj britannique, l'histoire de l'empire romain devint un paysage mental de référence ».

Robert Irwin, For Lust of knowing. The Orientalists and their enemies, Londres, Allen Lane, 2006, p. 159. 
Je souhaite montrer dans cet article que l'analogie entre les empires romain et britannique rencontre l'analogie entre la Bretagne médiévale et l'Inde coloniale au moment précis des « gémissements des Bretons »: autrement dit, entre le départ des Romains de la Bretagne au début du $\mathrm{V}^{\mathrm{e}}$ siècle et l'arrivée des Angles et des Saxons au vI $\mathrm{v}^{\mathrm{e}}$ siècle. Cet intervalle est le point de suture entre deux trajectoires de conquête, qui, grâce au trope de la translatio imperii et studii, ouvre la voie au « début du récit de la nation »; mais il souligne aussi, ce faisant, le « moins dans l'origine » : la mise en œuvre de cette téléologie britannique en a effacé toute trace de passé celtique.

\section{Normands et Saxons dans le Bengale du XVIII ${ }^{\mathrm{e}}$ siècle}

Le 18 juin 1822, le Seizième régiment royal de dragons légers fit voile pour Calcutta à bord de la Marquise d'Ely. Pendant le voyage, les officiers découvrirent avec enthousiasme Ivanhoé, qui venait d'être publié. Peu après, en décembre 1822 ou en janvier 1823, une fête en l'honneur d'Ivanhoé fut organisée à Calcutta. Un des participants, un certain Capitaine Luard, doté de toutes les qualités attendues d'un jeune officier, tint non seulement son rôle dans les préparatifs, mais réalisa également une esquisse représentant l'assemblée des dignitaires et de leurs épouses, portant le costume des principaux personnages du roman ${ }^{8}$.

Transportés de la métropole à la colonie, ces officiers du Seizième régiment ne trouvèrent pas meilleure distraction que de se déguiser en ce que Macaulay appelle «nos progéniteurs saxons et normands ${ }^{9}$ ». Cette anecdote rend compte de manière significative de l'état d'esprit qui régnait au début du XIX ${ }^{\mathrm{e}}$ siècle à Calcutta, ainsi que des préoccupations subliminales de ceux qui bravèrent les océans pour s'emparer des premières terres impériales. Il n'y a rien d'étonnant à ce que le « désir des origines ${ }^{10} »$, incontestablement exacerbé par le voyage vers l'Inde, se soit articulé de manière convaincante avec cet entrelacement idéalisé de Saxons et de Normands que met en scène Ivanhoé. Sans compter les résonances que le thème général du roman de Walter Scott (la relation de concordance qu'entretiennent la race et la langue avec les questions de pouvoir) a pu susciter chez les officiers après qu'ils ont débarqué au Bengale : moins évidentes peut-être, elles sont implicitement présentes dans cette reprise d'Ivanhoé à Calcutta. 


\section{Translations analogiques}

Au cours des années 1770-1790, les érudits britanniques commencèrent à produire toute une série de grammaires, de dictionnaires, de livres de classe et de traductions de différentes langues indiennes. Cette vague d'activité linguistique démarra au moment où les Britanniques purent avoir accès aux revenus du Bengale, à la suite de la victoire britannique à la bataille de Plassey en 1757, sous le commandement de Robert Clive. De facto, Plassey avait rendu les Britanniques maîtres du Bengale, point d'ancrage à partir duquel ils pourraient rapidement étendre leur emprise sur le reste de l'Inde. De manière stratégique, les vainqueurs firent main basse sur les langues qu'ils découvrirent, des plus élevées (le sanskrit, l'arabe et le perse) aux plus prosaïques (le bengali parlé et le urdu/hindustani). Une véritable armée de linguistes se mit en ordre de bataille pour réaliser cette ambition. L'orientaliste était né : parmi ceux qui incarnèrent le phénomène figurent Sir William Jones, fondateur de la Société asiatique et premier à percevoir des ressemblances dans les structures du sanskrit, du perse, du latin et du grec, mais aussi quelqu'un comme Nathaniel Halhed, auteur de la première grammaire bengalie ${ }^{11}$. Produire et consolider cet appareil linguistique permit d' « établir une formation discursive, de définir un espace épistémologique, de créer un discours (l'orientalisme) et de convertir les formes indiennes du savoir en objets européens ${ }^{12}{ }^{2}$.

C'est dans ce contexte qu'il convient d'appréhender la publication en 1787, par John Borthwick Gilchrist, du Dictionnaire anglais/hindustani, l'hindustani étant l'une des langues parlées au nord de l'Inde. Pour faire connaître à ses lecteurs l'histoire linguistique de l'Inde, Gilchrist, chirurgien dans l'armée britannique, formule à propos de l'hindustani les remarques suivantes dans la préface de son Dictionnaire :

Avant les incursions des musulmans et leur colonisation du territoire, l'hinduwee (ou hindooee) était à l'Inde ce que l'hindustani est aujourd'hui à l'Hindustan. [...] Avec diverses modifications, cette langue ancienne est à l'Hindustan exactement ce que le saxon était à l'anglais avant la conquête normande. Et l'hindustani n'est en réalité rien de plus que l'hinduwee infiltré par l'arabe et le perse après la victoire des envahisseurs musulmans. Il entretient presque en tout point avec sa base originelle les mêmes relations qu'entretient aujourd'hui avec sa propre source l'anglais qui naquit de son parent saxon, brouillé par l'afflux du français et d'autres langues continentales ${ }^{13}$.

Ce commentaire tortueux constitue l'une des premières utilisations du Moyen Âge par les Britanniques pour éclairer le présent indien par 
analogie. «Exactement », « rien de plus que », « presque en tout point» : par ces termes, Gilchrist met à bas les différences entre l'Angleterre médiévale et l'Inde contemporaine, et fait entrer de force son argument dans les contours d'un raisonnement analogique. Que ces comparaisons soient ou non exactes ou valides historiquement importe peu. L'essentiel, c'est que l'analogie est utilisée ici comme un outil rhétorique en vue de dégager un nouvel espace étymologique, qu'elle mobilise à cette fin le passé médiéval de la Bretagne.

Là où la métaphore et la comparaison impliquent un horizon limité et statique, l'analogie couvre un champ plus large. Une mise en regard métaphorique du Moyen Âge européen et de l'Inde peut, par exemple, conduire à affirmer que « les langues indiennes en sont [encore] au stade du Moyen Âge ». Transformée en comparaison [simile], cette phrase donnerait : " les langues indiennes sont [encore] comme les langues du Moyen âge ». Par contraste, les analogies comparent des systèmes et des mouvements et permettent ainsi à celui qui les utilise de produire une sorte de calcul sémiotique. L'analogie de Gilchrist ne compare pas simplement le saxon à l'hindou et le normand au musulman ; bien plutôt, elle enferme dans la même arène discursive les relations qui unissent le saxon et le normand d'une part, l'hindou et le musulman de l'autre. En faisant du développement de l'hindustani un décalque de celui de l'anglais à partir de son "parent saxon ", elle applique aux langues et aux cultures qui les alimentent un point de vue interprétatif singulier, qui a pour foyer l'idée répandue de la conquête comme dynamo du changement culturel.

L'Hindustan - le pays des hindous ou de ceux qui habitent au-delà des rives de l'Indus - est la forme persane de l'arabe Al-Hind. L'hinduwee et l'hindustani sont deux versions du langage parlé dans une bande de terre qui traverse le nord de l'Inde : elles sont identiques d'un point de vue grammatical, mais sont dotées de composants lexicaux différents. L'hinduwee (ou hindi) a puisé une grande partie de son vocabulaire dans les inflexions tardives du sanscrit. L'hindustani contenait une forte proportion de mots dérivés du perse, de l'arabe et du turc, autant de langues introduites par les vagues successives de musulmans venus d'Asie centrale et entrés en Inde par le détroit de Khyber à partir du XII ${ }^{\mathrm{e}}$ siècle pour une multitude de raisons, parmi lesquelles le pillage, la conquête, le commerce et la propagation pacifique de l'islam. Gilchrist choisit de mettre au premier plan les dimensions militaires et expansionnistes du 


\section{Translations analogiques}

développement de l'hindustani en le comprenant — et en l'expliquant - par analogie avec la conquête normande. Annonçant étrangement Ivanhoé, cette analogie entre les « invasions musulmanes » et la conquête normande donne à lire cette dernière comme rupture culturelle préalable à un rapprochement linguistique.

Cette interprétation apparaît clairement lorsque Gilchrist demande à son lecteur de se retourner sur le passé pour observer l'Angleterre anglo-normande :

Marquons ici une terrible pause, pour revenir à l'Angleterre telle qu'elle fut du temps de Guillaume le Conquérant et bien après lui. Avonsnous complètement oublié que le perse est à présent à l'hindustanee ce que le français normand était à l'anglais misérablement opprimé de cette sombre période ? Et pouvons-nous malgré tout continuer à encourager, à cultiver et à étendre parmi nous, grâce à des lois et des règlements britanniques bienveillants, l'acquisition d'un emblème d'esclavage, étranger et parfaitement odieux, et la soumission, poussée presque jusqu'à l'éradication totale, des deux dialectes locaux issus de la langue générale intermédiaire qu'est l'hindustani, où l'hindou non officiel lui-même a disparu depuis longtemps en rencontrant sur sa route le fier musulman, pour unir les conquis et les conquérants de l'Inde ? (p. XxvII)

La structure analogique permet ici au «fier musulman » de s'incorporer le normand, à l'« anglais misérablement opprimé » de se fondre dans l'hindou ; et la langue étrangère, odieux emblème d'esclavage et de soumission, en vient à décrire aussi bien le français que le perse. Gilchrist campe ensuite une cour de justice anglo-normande peuplée de magistrats et de magistrats uniquement francophones, d'officiers indigènes et d'agents subalternes bilingues français-anglais, et de «simples gens du peuple [anglophones]» (ibid.). Appelés à se prononcer sur des affaires dont le détail leur est traduit du vernaculaire par leurs subordonnés bilingues, les juges anglo-gaulois francophones ne peuvent que se tromper; de même en Inde, souligne Gilchrist, un juge britannique qui excellerait en persan mais ignorerait l'hindustani pourrait se faire une idée fausse d'un innocent sujet britannique appelé à comparaître.

Par cette fastueuse mise en scène du monolinguisme de l'élite, Gilchrist vise à convaincre ses lecteurs anglais - et les utilisateurs potentiels du Dictionnaire - de la nécessité de connaître la langue vernaculaire locale. Son récit fait toutefois moins appel aux « brahmines rusés » bien connus aujourd'hui, ces intermédiaires bilingues qui menèrent les orientalistes en 
bateau, qu'à une vision particulière de l'Angleterre d'après la Conquête, et à ses implications politiques. Les épithètes qu'il applique au normand et au saxon renvoient à des versions de la théorie du Joug normand marquées par le radicalisme, et qui, tenues sous le boisseau depuis la période des Niveleurs et des Bêcheux ${ }^{14}$, firent leur grand retour dans le dernier quart du XVIII ${ }^{\mathrm{e}}$ siècle, catalysées par les révolutions américaine et française ${ }^{15}$.

\section{Normands, Mongols et despotes orientaux}

Publié anonymement en 1771, l'Essai historique sur la Constitution anglaise, préconisant « la réconciliation entre la Grande-Bretagne et ses provinces lointaines », attribuait à Alfred le Grand, roi des AngloSaxons, l'institution du bicaméralisme parlementaire, du jugement par jury et du gouvernement démocratique. « Cela que les Saxons établirent est d'ordre véritablement constitutionnel, mais ceci que les Normands ont instauré ne s'y rapporte en rien, et participe d'un état d'esprit tyrannique $^{16} »$. Largement diffusé, l'Essai rendait compte de la formation de la Constitution anglaise selon un schéma similaire à celui envisagé par Gilchrist et consorts pour la formation de la langue anglaise. De même que celle-ci est née d'un mélange de saxon et de français, de même la Constitution anglaise

consiste en un mélange des anciennes, ou premières, institutions et des nouvelles, ou de celles qui ont été installées par William I ${ }^{\mathrm{er}}$ au moment de ce qui a communément été appelé la Conquête (et depuis lors). Ces deux formes de gouvernement sont diamétralement opposées : l'une s'appuie sur le principe de la liberté, l'autre sur les principes de l'esclavage ; il n'est pas étonnant qu'elles se livrent une guerre sans trêve. L'une, en effet, est fondée sur les droits naturels de l'humanité, qu'exprime l'exercice constant, reconduit chaque année, du pouvoir électif ; tandis que l'autre repose sur la domination despotique d'un seul homme. (p. 8)

L'expression «droits naturels » resurgira quelques années plus tard sous la très radicale plume de Thomas Paine : son Sens commun raillera une monarchie fondant ses prérogatives sur les actes illégitimes d'un «bâtard français flanqué d'une clique de bandits armés ${ }^{17}$ ». La manière dont Paine considérait l'Angleterre d'avant la Conquête n'était pas plus flatteuse ; cependant, pour ceux qui tenaient des positions un peu moins 


\section{Translations analogiques}

radicales que les siennes, l'insistance sur le Joug normand laissait percer une tendance tacite à la sympathie et à l'admiration envers les « libres Anglo-Saxons ».

L'année où parut l'Essai historique, l'historien français Augustin Thierry puisa dans le corpus littéraire afin de démontrer l'oppression des Saxons par les Normands et, par suite, préconiser le rétablissement des premiers. Pour mieux souligner l'inégalité des Saxons, leur langue est reléguée au rang de «partie de la nation la plus pauvre et la plus grossière », tombée « autant au-dessous du nouvel idiome anglo-normand, que celui-ci était au-dessous du français, langage de la $\operatorname{cour}^{18} »$. La résurgence de la langue anglaise au $\mathrm{Xv}^{\mathrm{e}}$ siècle voit disparaître l'inégalité linguistique qui, «avec l'inégalité de condition sociale, avait marqué la séparation des familles issues de l'une ou de l'autre race ${ }^{19} »$. Thierry use d'autres analogies pour souligner « la ressemblance entre l'état des Grecs sous les Turcs et celui des Anglais de race sous les Normands, non seulement pour ce qu'il y a de matériel dans l'asservissement, mais pour la forme particulière que revêt l'esprit national au milieu des souffrances de l'oppression, pour les instincts moraux et les croyances superstitieuses qui en naissent, pour la manière de haïr ceux qu'on voudrait et qu'on ne peut vaincre, et d'aimer ceux qui luttent encore lorsque la masse courbe la tête ${ }^{20} »$.

Que Thierry fasse référence à l'Empire ottoman en dit long sur la manière dont s'est construit le parallèle entre les musulmans qui envahirent l'Inde et les Normands qui envahirent l'Angleterre anglo-saxonne. Clé de voûte de ce parallèle : la figure du despote oriental, antithèse d'un modèle qui finirait par constituer les idéaux révolutionnaires de liberté et d'égalité ${ }^{21}$. Tandis que, sur la foi d'un islam commun aux Mongols et aux Ottomans, la thèse du despotisme oriental se diffusait vers l'est de l'Inde, le credo de défense des opprimés adopté par le radicalisme au sens large facilita sa progression vers l'ouest, direction l'Angleterre d'après la Conquête. Les termes utilisés dans les écrits radicaux de la fin du XVIII ${ }^{\mathrm{e}}$ siècle pour évoquer la domination de Guillaume le Conquérant — l'esclavage, la tyrannie et le despotisme - rappellent les parallèles établis par Gilchrist entre les dominations normande et musulmane. C'est dire que les analogies associant l'Angleterre médiévale et l'Inde n'eurent pas seulement à voir avec la disjonction entre passé médiéval et modernité, telle qu'elle fut introduite à l'époque des Lumières : un étalonnage supplémentaire survint lorsque leur fut appliqué le schéma de l'indigène 
opprimé face à l'envahisseur oppresseur, puisé lui aussi aux sources multipes des Lumières.

L'analogie formulée par Gilchrist montre bien qu'au tournant du siècle, un système à deux niveaux vit le jour pour comparer l'Inde et le Moyen Âge : certains aspects de l'économie politique et culturelle indienne étaient comparés à l'époque d'avant la Conquête, celle des « libres Anglo-Saxons », tandis que d'autres étaient comparés aux temps d'après, dominés par des Anglo-Normands féodaux et despotiques. Bien qu'un grand nombre de ceux qui traitèrent de l'Inde en ces termes fussent des orientalistes au sens classique, c'est-à-dire des « linguistes [proto-comparatistes] », d'autres, comme
"L'Union [européenne] ressemblera à un empire, mais ce ne sera pas le type d'empire que les eurosceptiques redoutent tant. L'UE n'est pas en train de devenir un superÉtat projetant son autorité sans cesse grandissante sur tout le continent européen et au-delà. Ce qu'elle devient, c'est une entité politique polycentrique, qui pénètre son environnement plutôt qu'elle ne le contrôle. Il est probable que la multiplicité et le chevauchement des institutions militaires et policières demeurent la norme. Entre cette UE et la puissance impériale des États-Unis d'aujourd'hui, ou de la Grande-Bretagne au xIxe siècle, il y a un puissant contraste. De fait, l'empire auquel l'UE ressemble nous fait remonter plusieurs siècles en arrière. Les caractéristiques de son système de gouvernement (pluralité des niveaux, flou des frontières et formes adoucies de projection de son autorité vers l'extérieur) l'assimilent au système que nous avons connu au Moyen Âge, avant l'émergence des États-nations et du capitalisme ».

Jan Zielonka, Europe as Empire. The Nature of the enlarged European Union, Oxford, Oxford University Press, 2006, p. 1.

le colonel James Tod, résident britannique au Rajasthan, étaient animés par une imagination analogique qui informait jusqu'à leurs documentaires de voyage ou leurs récits historiques. Les Annales et Antiquités du Rajasthan, cet ouvrage extrêmement populaire publié par Tod entre 1829 et 1832 et écrit, comme le souligne le titre, dans la lignée de Percy, Ossian et Walter Scott, fait du Rajasthan une véritable terre de Highlands, regorgeant de bardes, de cairns et de ménestrels ${ }^{22}$. Nombreux sont, dans cette tapisserie, les fils tendus entre les brahmines et les moines anglosaxons, les États des Rajputs et l'heptarchie saxonne, leurs adversaires moghols et les despotes ou les tyrans à la mode normande.

Bien qu'il n'use pas de l'analogie avec la même application méthodique, Tod partage avec des orientalistes comme Gilchrist, Halhed, 


\section{Translations analogiques}

Colebrooke, voire Jones, la même admiration pour une strate au moins de la langue et de la culture indienne : celle qui correspond en gros à l'Inde hindoue. Il partage également avec eux l'idée que certaines langues de l'Inde (le perse, l'arabe et le sanscrit) sont aussi « classiques » que le latin et le grec, alors que les langues parlées au quotidien dans les différentes régions du pays sont vernaculaires. Les orientalistes et leurs disciples ont utilisé le normand et le saxon, à l'instar du classique et du vernaculaire, comme des catégories épistémologiques pouvant, grâce à l'analogie, remettre de l'ordre dans l'épais massif de nouvelles données que l'Inde représentait pour eux. La relation particulière qui s'est alors développée entre la race, la langue et la conquête a coloré autant le passé anglais que le présent indien, ce qui, dans les écrits des orientalistes, visait à décrire davantage qu'à prescrire ${ }^{23}$.

\section{La romanitas et la renaissance du Bengale}

Au début du XIX $X^{e}$ siècle, après que la Compagnie orientale des Indes eut fait l'objet de plusieurs causes célèbres, tel le procès de Warren Hastings, le terrain linguistique du Bengale devint moins favorable ${ }^{24}$. Les questions touchant au devoir moral des Britanniques à l'égard des Indiens et à la responsabilité de la Compagnie furent placées au cœur du débat et de la pratique. L'entrelacement entre libéralisme, utilitarisme et empire produisit un mélange de ces questions avec le débat sur l'éducation de la population indigène ${ }^{25}$. Le choix de la langue d'instruction la plus appropriée devint alors un objet de litige ${ }^{26}$. Pour les orientalistes, forts d'un quart de siècle d'expérience linguistique, il convenait de choisir entre les langues indiennes «classiques » et les langues «vernaculaires » régionales. Leurs opposants, appelés anglicistes, considéraient au contraire toutes les langues indiennes comme un vecteur et un réceptacle de la superstition, de l'idolâtrie et d'une civilisation arrêtée dans son évolution. Animés par un mélange de conviction utilitariste et de zèle évangélique, ils se réclamaient de l'anglais comme d'un moyen privilégié d'instruction, retournant avec succès les arguments érudits des orientalistes contre ceux-ci.

Le Rapport sur l'éducation indienne (1835) de Thomas Babington (Lord Macaulay) est le manifeste le plus (tristement) célèbre de la position angliciste. Adressé au Comité général de l'Instruction publique en 
Inde, il détourne l'analogie orientaliste associant l'Angleterre médiévale et l'Inde contemporaine :

Si nos ancêtres avaient négligé la langue de Cicéron et de Tacite, s'ils avaient cantonné leur attention aux vieux dialectes de notre île, s'ils n'avaient rien imprimé ni enseigné dans les universités, hormis des chroniques en anglo-saxon et des romances en français normand, l'Angleterre serait-elle ce qu'elle est ? Ce que le grec et le latin étaient aux contemporains de More et d'Ascham, notre langue l'est au peuple de l'Inde. La littérature d'Angleterre vaut plus que celle de l'Antiquité classique. Je doute que la littérature sanscrite ait autant de valeur que celle de nos ancêtres saxons et normands ${ }^{27}$.

À l'instar de Gilchrist, Macaulay distingue l'(anglo-)saxon et le normand(-français), et assimile chaque période avec un genre spécifique de la production littéraire : la chronique et la romance. Il s'éloigne cependant de Gilchrist quand il évoque une troisième phase de développement linguistique et culturel, qui tranche aussi bien avec le saxon qu'avec le normand : celle de la Renaissance / Réforme, marquée par le renouveau de la langue de Cicéron et de Tacite. De même que l'Angleterre fut tirée des « ténèbres » médiévales par la lumière des classiques durant la Renaissance, de même l'anglais jouerait ce rôle en menant le Bengale, voire l'Inde tout entière, vers sa propre Renaissance. Ainsi put-on parler d'une « Renaissance du Bengale »: l'expression, qui consacre l'hypothèse angliciste, demeure courante encore aujourd'hui pour désigner la période d'activité littéraire qui suivit l'application de semblables postulats par William Bentinck, le gouverneur-général du Bengale.

Reprenant l'analogie orientaliste entre les Normands et les musulmans d'une part, les Saxons et les hindous d'autre part, Macaulay lui donne une nouvelle tournure : ce que les classiques étaient à l'Angleterre médiévale, l'Angleterre moderne l'est à l'Inde (contemporainequoique-restée-médiévale). Les évangélistes avaient eux aussi leur lecture du même argument : selon le missionnaire écossais Alexander Duff, «l'anglais occupe à présent la même place en Inde que le latins et le grecs en Europe à l'époque de la Réforme ${ }^{28} »$. Les deux interprétations renvoient au trope de la translatio imperii, et inversent, en réalité, l'utilisation plus ancienne qu'en faisaient les orientalistes eux-mêmes. Comme le rappelle Cohn, Nathaniel Halhed avait lui aussi comparé aux Romains les Anglais installés en Inde au XVIII siècle, mais dans un sens diamétralement opposé : « les Anglais maîtres du Bengale », écrit 


\section{Translations analogiques}

Halhed en 1778, ressentaient le besoin de s'approprier aussi la langue du pays, comme les Romains, «peuple d'érudition médiocre et de peu de goût, qui, après avoir conquis les Grecs, s'était consacrés à l'étude de leur langue ${ }^{29} »$. Ignorant la « Rome barbare » qui cadrait mal avec leur argumentation, les anglicistes préférèrent insister sur le transfert des manuscrits grecs et latins vers l'Europe après la chute de Constantinople et sur le renouveau érudit qui en découla, un renouveau à l'ombre duquel Rome s'était peu à peu hellénisée plusieurs siècles auparavant.

Somme toute, une fois reprise par les anglicistes, qu'ils soient utilitaristes ou évangélistes, l'analogie tourne à la téléologie : si le Moyen Âge, qui englobe l'Angleterre anglo-saxonne et celle de l'après-Conquête, constitua pour la langue anglaise une période comparable à celle que connaissaient alors les langues indiennes, ce n'était qu'une étape dans le développement général de l'anglais (et des Anglais) vers un idéal classique et moderne à la fois. La croyance selon laquelle toutes les langues vernaculaires devaient posséder une « littérature à elles » pour pouvoir progresser au sein du récit de la civilisation joua un rôle crucial dans la discussion. L'argument est formulé par un compagnon angliciste de Macaulay, Sir Charles Trevelyan, qui, comme Macaulay avec son Rapport, adressa son traité Sur l'éducation du peuple indien (1838) au Comité général de l'Instruction publique. Considérant la « formation d'une littérature vernaculaire comme l'ob-
« Provincialiser l'Europe ne traite pas de la région du monde que nous appelons Europe. Cette Europe, on pourrait dire que l'histoire l'a déjà provincialisée. Sud-Est moderne : c'est là que sont mes archives et mon site d'analyse. L'Europe que je cherche à provincialiser ou à décentrer est une figure imaginaire qui, sous forme de clichés et de raccourcis, demeure profondément ancrée dans certaines habitudes de la pensée ordinaire qui sous-tendent invariablement les tentatives faites par les sciences sociales pour soulever la question de la modernité politique en Asie de l'Est. Le phénomène de la "modernité politique" - à savoir la domination de ces institutions modernes que sont l'État, la bureaucratie et l'entreprise capitaliste - est tout simplement impensable, où que ce soit, sans un certain nombre de catégories et de concepts profondément enracinés dans la tradition intellectuelle, voire théologique de l'Europe. »

Dipesh Chakrabarty, Provincialiser l'Europe. La pensée postcoloniale et la différence historique (trad. de l'anglais (Inde, Australie, États-Unis) par Olivier Ruchet et Nicolas Vieillescazes), Paris, Éditions Amsterdam, 2009 [2000], p. 31-33). [...] De formation, je suis historien de l'Asie du 
jectif ultime vers lequel doivent être tendus tous nos efforts », Trevelyan affirme que « l'étude de l'anglais, et avec elle, l'apprentissage du savoir occidental, est la première étape à franchir pour pouvoir éclairer l'Inde ${ }^{30} »$. Le lien étroit qu'il établit entre la langue, la littérature et l'éducation libérale renvoie ici à une distinction lourde de sens entre deux types de vernaculaires : d'un côté, les langues à littérature, telles les langues modernes européennes; de l'autre, celles qui, de l'avis des anglicistes, en sont dépourvues, c'est-à-dire les langues de l'Inde.

Mu par le souci d'améliorer ces dernières, le traité de Trevelyan préconise de mettre en œuvre un processus graduel de changement culturel. Il en résume les linéaments tout en écartant les objections stylistiques que pourrait soulever un locuteur indien, eu égard au noyautage de ses vernaculaires par l'anglais :

Un argument aussi raffiné constitue la dernière étape dans la marche du progrès. C'est pour la langue un luxe absolu. Et dire qu'un Bengali ou un Hindustani pourrait être blessé dans sa sensibilité délicate par l'introduction de nouvelles idées, cela veut dire qu'on transfère sur une langue pauvre et informe des sentiments qui ne s'attachent qu'à une langue riche et cultivée. Ils ne pourront penser à préserver la pureté de leur langue que lorsqu'ils auront réglé leur vocabulaire scientifique et possèderont leurs propres chefs-d'œuvre. Quand ils auront un Milton ou un Shakespeare, ils ne nous demanderont pas de les guider en cette matière. (p. 122-123)

De ce passage nous pouvons déduire les cinq étapes suivant lesquelles une langue simplement vernaculaire peut devenir une langue à littérature :

1. Langue pauvre et informe, dépourvue de vocabulaire scientifique.

2. Vocabulaire infusé par la science, facteur de progrès et d'amélioration.

3. Apparition du Milton et du Shakespeare indigènes, production des premiers chefsd'œuvre.

4. Une langue riche et cultivée, pour finalement parvenir au :

« Vers 2050, plus tôt probablement, toute connaissance de l'ancienne langue aura disparu. Toute la littérature du passé aura été détruite. Chaucer, Shakespeare, Milton, Byron n'existeront plus qu'en versions novlangue. Ils ne seront pas changés simplement en quelque chose de différent, ils seront changés en quelque chose qui sera le contraire de ce qu'ils étaient jusque-là ».

George Orwell, 1984 (trad. de l'anglais par Amélie Audiberti), Paris, Gallimard, Folio, 1990 [1949], p. 80 . 


\section{Translations analogiques}

5. Luxe absolu de la langue : finesse, pureté et délicatesse stylistique.

Ce programme d'amendement des vernaculaires indigènes renvoie, fondamentalement, à un paradigme qui cristallise une imbrication particulière des histoires linguistique et politique de l'Angleterre. Le stade 1 peut ainsi être lu comme anglo-saxon, le stade 2 comme médiéval et le stade 3, marqué par les Milton et les Shakespeare, comme un équivalent de la Renaissance. Pendant la Renaissance, l'influence des classiques permit de perfectionner toutes les «langues modernes européennes [qui] étaient à un stade excessivement barbare, dénué d'élégance, de vigueur et même de clarté. [...] Par la suite, l'apparition d'exigences que les langues classiques étaient incapables de satisfaire a fait naître la littérature vernaculaire européenne » (p. 40-41). Trevelyan cite alors le Rapport de Macaulay pour étayer l'idée selon laquelle « dans son état actuel la littérature hindoue et musulmane ressemble de près, mutatis mutandis, à ce qu'était la littérature européenne avant l'époque de Galilée, de Copernic et de Bacon» (p. 57).

Au sein de ce schéma en cinq étapes, les distinctions entre le normand et le saxon ne suscitent plus l'attraction ou la répulsion que leur avaient values les oppositions entre les Anglo-saxons « libres » et l'Angleterre « despotique » de l'après-Conquête. Les radicaux critiques du Joug normand continuèrent à se faire entendre, mais ne se cantonnaient plus désormais au seul créneau de l'après-Conquête : c'est l'ensemble du Moyen Âge, vu comme féodal et pré-moderne, qui s'en trouvait noirci à leurs yeux. En version évangélique, le Joug normand féodal devint aussi le « Joug scolastique ». Ainsi, selon Duff, « quand vous discutez avec les brahmines, vous êtes transporté dans l'âge des ténèbres européen. Les distinctions minutieuses, les subtilités spéculatives... tout ce monde des facultés se rappelle avec force à votre mémoire ${ }^{31} \gg$. Dans le même temps, le crédit qu'accordait la pensée radicale à la période anglo-saxonne tendit à s'amenuiser. Certes, la distinction entre normand et saxon était toujours de rigueur, et demeurait, comme chez les orientalistes, calquée sur les distinctions entre périodes hindoue et musulmane de l'histoire indienne. Pourtant l'analogie avait changé de portée, ainsi qu'en témoigne clairement ce commentaire de Trevelyan :

Le système hindou de connaissance comporte assez de vérité pour que cette nation se soit hissée à son stade actuel de civilisation, et s'y soit maintenue des siècles durant sans retour en arrière ; et il comporte assez 
d'erreur pour l'avoir empêchée, tout au long de cette longue période, de connaître la moindre avancée sensible. [...] Le système mahométan de connaissance est supérieur à bien des égards et ressemble à celui que possédaient les nations européennes avant l'invention de l'imprimerie, ce qui le situe bien loin du savoir dont l'Europe est à présent dotée. (p. 83-85)

Le stade « médiocre » et le stade « légèrement meilleur », qui correspondent à l'Europe pré-renaissante, reprennent en écho l'analogie formulée par Gilchrist pour associer le normand et le musulman, le saxon et l'hindou, et opposer le normand et le saxon.

Le passé médiéval européen n'en diffère pas moins du présent vernaculaire indien en un aspect fondamental : il évolue au rythme de ce que Trevelyan appelle une «avancée sensible ». Par contraste avec cette vision d'une société hindoue arrêtée dans son progrès et avec celle, à peine plus positive, de la société musulmane (qui rejoint la manière dont John Stuart Mill considère l'Inde), la culture européenne, et particulièrement anglaise, est vue comme foncièrement évolutive et animée par un progrès constant :

Lorsque le latin était [...] le berceau des sciences, la langue anglaise n'avait pas atteint cette plénitude et cette exactitude dont elle peut aujourd'hui légitimement se prévaloir. Le style des écrivains vernaculaires n'était pas encore formé ; il restait faible, pédant et corrompu ; la composition en était à peine au stade de l'enfance et on ne comptait que peu d'auteurs. [...] À partir de la Réforme, l'anglais s'est peu à peu rapproché du magnifique état qu'il a atteint aujourd'hui et qui associe l'universalité, l'abondance et la beauté (p. 216).

De manière évidente, le trope de la translatio imperii a influencé le concept d' «avancée sensible » formulé par Trevelyan, mais la relation fonctionnait dans les deux sens. La prééminence que les anglicistes accordent aux classiques rejoint la manière dont on assimilait déjà les Anglais aux conquérants romains ; elle nourrit l'amalgame entre l'idée d'une Renaissance bengalie conduite par eux et la mise en parallèle, sur le plan politique, de la Rome impériale avec la Bretagne proto-impériale. Un article paru en 1845 dans la Calcutta Review affirmait ainsi : «nous avons été appelés [...] pour imiter les conquérants romains, lesquels ont civilisé et conquis les nations qu'ils ont soumises, [...] pour répandre une lumière victorieuse bien au-delà des terres où vola jamais l'aigle romain ${ }^{32} »$. De la même manière, Trevelyan clamait au Parlement que « nous ferons 


\section{Translations analogiques}

plus que les Romains », parce que les Britanniques ont deux avantages sur eux : la « liberté constitutionnelle » et la « chrétienté ${ }^{33} »$.

\section{Angoisses au cœur de l'Empire}

C'est au cours de la même discussion parlementaire que Trevelyan évoqua les "gémissements des Bretons » placés en épigraphe à cet article. S'appuyant sur la comparaison entre la Rome impériale et l'Empire britannique, Trevelyan s'interroge sur la manière dont réagiraient les Indiens si les Britanniques renonçaient à la responsabilité qu'ils ont conçue à leur endroit : celle de les mener à la « modernité » à l'aide de la langue anglaise. Or, les Bretons que nous avions laissés gémissant derrière les Romains, eux dont les gémissements annonçaient aussi l'arrivée des Angles, des Saxons et des Jutes invités par un Vortigern assiégé $^{34}$, sont ceux-là mêmes qui furent les précurseurs et les ancêtres des Britanniques — équivalence à laquelle l'Acte d'Union de 1800 confère un relief tout particulier ${ }^{35}$. Autrement dit, les «Bretons gémissants » représentent, sous forme de message codé, à la fois les colonisateurs et les colonisés. À ce stade du dispositif rhétorique mis en œuvre par Trevelyan, l'analogie semble corseter de manière presque trop serrée un schéma généalogique et territorial particulièrement complexe.

Quand on le réquisitionne au service des origines, le parallèle entre les empires romain et britannique renvoie inévitablement, quoique non sans réticences, au moment où les Romains et les Saxons s'associèrent pour former un couple parental idéal(isé), à la fois politique et généalogique, mais aussi au moment où le passé celtique de la Bretagne fit son intrusion, livrant aux regards le «moins dans l'origine ». En 1851, près de trente ans après avoir décrit la fête d'Ivanhoé à Calcutta, le capitaine Luard publia à compte d'auteur une histoire illustrée du costume militaire britannique $^{36}$. Ses trois premières esquisses reconstituent le costume militaire de la Bretagne romaine, saxonne et normande, et la dernière fait voisiner des soldats britanniques contemporains avec un Indien montré dans une attitude de soumission. La succession linéaire des illustrations invite à interpréter l'histoire de la Bretagne comme une progression téléologique depuis le statut d'ancienne colonie romaine jusqu'à la puissance impériale, exactement comme le soldat britannique romain qui précède le Saxon incarne les strates proto-coloniales amalgamant, 
au passé anglo-saxon, les généalogies putatives du sang et du pouvoir. Les commentaires que formule Luard à propos des habitants celtiques des îles britanniques sont plus révélateurs encore : «leur costume est très mal connu, c'est pourquoi nous ne tentons pas de le dessiner » (p. 1). Dans cette non-image qui hante la téléologie visuelle de Luard est inscrite la même aporie que dans l'évocation par Charles Trevelyan des « gémissements des Bretons ».

L'insistance d'Isaac Disraeli sur le fait que « les aborigènes de cette île ont disparu, mais que leur nom nous est encore attaché ${ }^{37} \gg$ est tout aussi importante. Le fossé entre les Bretons et les Britanniques, que creuse « l'acte de disparition » des soi-disant aborigènes, est comblé par la déclaration ultérieure de Disraeli, selon lequel « les Anglo-Saxons sont devenus nos progéniteurs et le saxon notre langue maternelle ». Les antécédents juifs de Disraeli révèlent en pleine lumière les motifs qui l'ont conduit à élaborer cette argumentation stratégique, dont un autre exemple, antérieur, est repérable chez Alexander Duff, dans un discours destiné à lancer les Anglais à l'assaut des Indiens. Dans un moment extraordinairement poignant, celui qui fut à la fois un missionnaire guindé et un colonel écossais exemplaire se lamente soudain de la disparition du gaélique sous l'effet de l'anglicisation : "C'est là pour moi une expérience douloureuse, car le gaélique est ma langue maternelle ${ }^{38}$ ». Après que les anglicistes eurent gagné la bataille de la langue, il était devenu moins urgent de souligner ainsi l'anglicité en se réclamant de la langue anglaise aux dépens d'autres filiations. En lieu et place, les « libres Anglo-Saxons » firent leur réapparition : ancêtres grossiers, mais vigoureux, d'un pouvoir rayonnant sur les mers, à l'image du destin que les analogies du XVIII' siècle, en l'assimilant à l'Empire romain, lui avait promis. Cette réintégration des Anglo-Saxons au sein de la manœuvre généalogique marque en filigrane l'impérialisme victorien — mais aux dépens, peut-être, du fragile équilibre analogique établi auparavant entre la Rome et la Grande-Bretagne impériales.

Le même court-circuit discursif traverse l'œuvre d'Edward Freeman, professeur à Oxford, historien de la Constitution et de la Conquête normande dans la seconde moitié du XIX ${ }^{\mathrm{e}}$ siècle. Ses travaux manifestent ostensiblement à quel point la confortable assurance de l'impérialisme victorien, alors en plein épanouissement, cajola le regain d'intérêt dont la question des origines anglo-saxonnes était l'objet. Son analyse des institutions politiques anglaises souligne que celles-ci « descendent de 


\section{Translations analogiques}

manière presque ininterrompue de la souche teutonne primitive », qu'elles ont « cru au sein du même corps » que « les traditions et coutumes grossières des successeurs de Hengest et Cerdic », sans que le «moindre fossé les sépare de l'assemblée du Witan du Wessex qui confirma les lois d'Alfred, et de celle, beaucoup plus ancienne, qui éleva l'échevin Cerdic au rang de roi ${ }^{39}$ ». Quant à la conquête normande, loin d' « étouffer ou d'éteindre le Vieil Esprit anglais », « elle le tira vers une forme plus précise et plus antagonique », transformant les « Normands conquérants » en « une bande de nobles prosélytes », après qu'ils se furent « gorgés » de « l'air » démocratique « de l'île libre ${ }^{40}$ ». Cette ancestralité anglo-saxonne, à la capacité d'absorption universelle, devient ainsi le fondement d'une anglicité robuste et proliférante, qui pour Freeman inclut catégoriquement la notion de britannicité impériale ${ }^{41}$.

Cette manière de lire le passé anglo-saxon à travers le filtre du présent impérial n'est guère éloignée de celle de l'auteur anglo-saxoniste John Mitchell Kemble, dont Freeman connaît et cite l'œuvre. Pour Kemble, le présent impérial lui-même ne saurait être égalé à l'avènement saxon :

Familiers que nous sommes des audacieuses entreprises navales qui ont vu notre drapeau flotter sur toutes les mers, battant à tous les vents de la surface terrestre, nous ne pouvons contempler sans surprise et admiration ces rudes marins qui partout fourmillaient, traversaient chaque océan, couvraient chaque estuaire, chaque baie, se jetant à terre dès que le rivage promettait du butin ${ }^{42}$.

En écho au jugement de Kemble, Freeman décrit l'Avènement saxon comme " l'arrivée d'une colonie envoyée au moment où notre race était encore dans un état de saine barbarie ${ }^{43} \gg$. Comme l'implique le mot «colonie », le passé et le présent, pour Freeman plus que pour Kemble, se mêlent typologiquement comme s'ils figuraient une destinée manifeste ${ }^{44}$. Aux termes du récit de Freeman, la prise de la Bretagne celtique par les Angles, les Saxons et les Jutes mime les aventures coloniales de l'Angleterre en Inde, en Afrique et aux antipodes : " nous avons gagné un pays pour nous-mêmes et nous avons grandi, peuple nouveau dans un pays nouveau ». En mettant l'accent sur la pureté raciale, il estompe sans accroc les siècles qui séparaient peurs anciennes et nouvelles, hantises du métissage et de la contagion : " disjoints de l'ancienne souche, préservés du mélange avec les autres, nous avons cessé d'être germains, sans pour autant devenir bretons ou romains ». 
Cette affirmation de pur anglo-saxonisme a des implications et des motivations plus larges. Freeman lit en effet la transformation de la Bretagne en Angleterre pendant la période anglo-saxonne non pas comme une teutonisation (comme le faisait le germanophile Kemble ${ }^{45}$ ), mais, conformément à ses propres prédilections, comme une anglicisation globale. Cette « anglicité » embryonnaire efface également les filiations raciales entre les Anglo-Saxons et les Saxons continentaux — ce mélange de barbares qui étaient auparavant regroupés sous la dénomination de « gothiques » divers et variés. La manière dont Freeman considère la « parenté gothique » oscille entre l'oubli et le souvenir : son insistance sur le fait que «nous avons cessé d'être germains » est assortie ailleurs d'une reconnaissance de la «famille teutonne » - lorsqu'il examine l'influence romaine, unique en son genre, dans la civilisation des hordes barbares, celles-ci ayant, selon le mot de Trevelyan, «triomphé des armées de Rome, avant de céder à ses $\operatorname{arts}^{46}$ ». Jeté à « ceux qu'elle a conquis », comme à « ceux qui l'ont conquise » (les Goths, qui restaurèrent « ses structures matérielles »; les Francs, qui restaurèrent «son autorité politique »), ce sort permet de disculper les cousins « barbares » des Anglais, tout en ajoutant à la gloire de Rome ${ }^{47}$.

Le développement d'un discours de l'indo-européanisme, et les nouvelles structures de parenté qu'il postule, autorisent aussi - ou peut-être contraignent - Freeman à associer « les Grecs, les Romains et les Teutons [qui] chacun leur tour, se démarquent des autres nations de la famille aryenne ${ }^{48} »$. L'utilisation par Freeman des structures de parenté indo-européennes est alliée à un redéploiement du trope de la translatio imperii. Les Teutons, les Grecs et les Romains, « chacun leur tour, ont atteint en pouvoir aussi bien qu'en civilisation le plus haut stade possible à leurs époques respectives, et chacun a transmis son propre patrimoine pour qu'il soit enrichi par des successeurs qui étaient à la fois des conquérants et des disciples ». La translatio imperii sépare le bon grain de l'ivraie au sein de la fraternité aryenne qui, sinon, juxtaposait de manière embarrassante l'impérialiste et le sujet. La chrétienté ellemême est lue comme une transmission du flambeau de la civilisation : « si le monde aryen européen a appris ses arts et ses lois d'un frère aîné, c'est de l'étranger sémite qu'il a appris sa foi. [...] Mais cette foi sémite, bannie de sa patrie sémite, est devenue le symbole de l'autorité romaine : qu'il s'agisse du Christ ou de César, leur emprise a fini par revêtir la même signification ${ }^{49}$ ». 


\section{Translations analogiques}

Le thème récurrent des travaux de Freeman est, in fine, celui d'une compétition entre différents groupes, apparus avant aussi bien qu'après Rome, pour conquérir le cœur et l'esprit de celle-ci. Son idée d'un lignage purement anglo-saxon des Anglais rend toutefois problématique la généalogie politique qui sous-tend cette thématique : si « la Bretagne est un pays qui a cessé d'être romain avant que ses conquérants teutons aient posé le pied sur son territoire », il s'ensuit que les Anglais n'ont en eux « aucun véritable élément romain ${ }^{50} »$. La translatio imperii accentue cet embarras plutôt qu'elle ne vient l'adoucir :

Quel que soit l'élément romain que nous avons en nous, nous ne le devons pas à une transmission directe depuis l'Empire antique, mais à notre conversion par les missionnaires romains, au fait que nous ayons été conquis à la fois par des guerriers et par des hommes de loi parlant le latin, et à l'esprit d'imitation qui a apporté aux seigneurs de notre île des titres empruntés aux Césars du continent ${ }^{51}$.

Le lignage intellectuel et politique dont Freeman se réclame avec véhémence trahit sa claire conscience de la disjonction généalogique entre, d'un côté, les Britanniques en tant qu'Anglo-Saxons et, de l'autre, l'Empire romain, patron suivant lequel la construction de l'Empire britannique était conçue. Recouvrant rapidement son aplomb, Freeman affirme :

Il y a trois foyers à notre peuple : la vieille Angleterre des Eider et des Slei, l'Angleterre plus récente que nous avons créée pour nous-mêmes dans le monde insulaire de la Bretagne, et la toute nouvelle Angleterre que nous avons étendue sur les îles et les continents de par l'Océan. Et en vérité, nous étions investis d'une mission, mais d'une mission qui ne se confond pas avec celle dont nos aïeux avaient été investis dans le cours général de l'histoire européenne ${ }^{52}$.

L'espace de la disjonction analogique rend ainsi nécessaire, et même nourrit, la croyance en la destinée manifeste de l'anglicité et en la mission impériale qui lui est échue, en tant qu'héritière et concurrente à la fois d'une romanitas transcendante : « Où que les hommes évoquent sa langue, où qu'ils révèrent sa loi, où qu'ils professent la foi qu'ont apprise d'elle l'Europe et les colonies européennes, il y a encore et toujours Rome ${ }^{53}$ ». Cette conviction, pourtant, demeure striée par la trace persistante des Celtes, qui «en leurs nom et langue propres, ne subsistent plus que çà et là dans les recoins ${ }^{54}$ », mais avec suffisamment d'énergie pour que Freeman ne puisse les ignorer. 
Par son incapacité finale à éliminer cette "poussière dans l'œil », Freeman, historien de l'Empire en son plein midi, n'est pas sans ressembler à un autre Anglais de l'époque plus ancienne du Raj, Reginald Heber, le premier évêque anglican de Calcutta. Plus connu pour ses Récits d'un voyage en Inde du Nord que pour ses poèmes, Heber, dans son fragmentaire « Morte D'Arthur» (1810-1820), capture pour ainsi dire dans l'ambre un instant éphémère, lorsque la vision de robustes Saxons transmués en imperators en toge se trouve soudain éclipsée par la nostalgie des jours perdus :

Quand du règne d'Arthur à la pompe chevaleresque,

Les somptueux festivals me reviennent en mémoire,

Quand je songe à toutes ces gloires celtiques,

Dissoutes, hormis le lai du ménestrel en ses rares vestiges,

Et dispersées en un courant d'oubli,

(Tant la lame des Saxons déchirait mortellement les plaies)

Force m'est de regretter cette périlleuse décrépitude,
"Et à ces mots, mon esprit suivant le cours des vicissitudes, qui ont tour à tour transmis le sceptre du monde à des peuples $s i$ différens de cultes et de mœurs, depuis ceux de l'Asie antique jusqu'aux plus récens de l'Europe, ce nom d'une terre natale réveilla en moi le sentiment de la patrie ; et tournant vers elle mes regards, j'arrêtai toutes mes pensées sur la situation où je l'avais quittée $\left.{ }^{*}\right)$.

Je me rappelai ses campagnes si richement cultivées ses routes si somptueusement tracées, ses villes habitées par un peuple immense, ses flottes répandues sur toutes les mers, ses ports couverts des tributs de l'une et de l'autre Inde; et comparant à l'activité de son commerce, à l'étendue de sa navigation, à la richesse de ses monuments, aux arts et à l'industrie de ses habitants, tout ce que l'Égypte et la Syrie purent jadis posséder de semblable, je me plaisais à retrouver la splendeur passée de l'Asie dans l'Europe moderne : mais bientôt le charme de ma rêverie fut flétri par un dernier terme de comparaison. Réfléchissant que telle avait été jadis l'activité des lieux que je contemplais : Qui sait, me dis-je, si tel ne sera pas un jour l'abandon de nos propres contrées ? Qui sait si sur les rives de la Seine, de la Tamise ou du Sviderzée, là où maintenant, dans le tourbillon de tant de jouissances, le cœur et les yeux ne peuvent suffire à la multitude des sensations ; qui sait si un voyageur comme moi ne s'asseoira pas un jour sur de muettes ruines, et ne pleurera pas solitaire sur la cendre des peuples et la mémoire de leur grandeur?»

C. F. Volney, Les Ruines, ou Méditation sur les révolutions des empires, Paris, Parmantier / Froment, 1826 [1791], p. 9-10 (souligné dans l'original).

(*) En 1782, à la fin de la guerre d'Amérique. (Note originale) 


\section{Translations analogiques}

Et, sans plus d'égards pour ma race, j'en viens presque à déplorer

Que jamais navire vent du nord n'ait le rivage de Logrian défloré55.

Cette vision fugitive (le colonisateur déplorant presque la périlleuse décrépitude infligée par la lame mortelle) ouvre un moment rare, où les structures du pouvoir le cèdent à la conscience soudaine d'une perte culturelle, en tant que perte d'humanité. Moment, aussi, de critique du médiévalisme impérial, qui des décombres même du déracinement extrait un humanisme résilient : paradoxale survivance qui simultanément atteste, loin du choc des civilisations, les transformations et la rédemption rendues possibles par la translation des cultures.

\section{Traduit de l'anglais par Laurence Marie}

1. Voir Norman Vance, The Victorians and Ancient Rome, Oxford, Blackwell, 1997. À propos de cette discussion parlementaire, voir les Rapports des comités, dans Parliamentary Papers, novembre 1852-août 1853, t. 32, appendice N ( « General Report on Public Instruction in the Lower Provinces of the Bengal Presidency for 1843-44»), p. 177.

2. Les « Codes du Menu » sont le Manusmriti sankrit, traité védique sur les coutumes hindoues qui fut la principale source de la codification de la loi hindoue par les Anglais.

3. Michael Winterbottom (éd. et trad.), Gildas: The Ruin of Britain and Other Works, Londres, Phillimore, 1978, «De excidio et conquestu Britanniae 20.1 », p. 95 : «Agitio ter consuli gemitus Britannorum. Et post pauca querentes : "repellunt barbari ad mare, repellit mare ad barbaros; inter haec duo genera funerum aut iugulamur aut mergimur.” Nec pro eis quicquam adiutorii habent » (« À Aetius, trois fois consul : les gémissements des Britanniques ». L'auteur poursuit ainsi sa plainte : «Les barbares nous ont repoussés vers la mer; et la mer nous repousse vers les barbares ; nous avons le choix entre deux types de morts : la noyade ou le massacre ». Ils n'obtinrent aucune aide). Voir aussi Nicholas Howe, «Anglo-Saxon England and the postcolonial void », dans Ananya Jahanara Kabir, Deanne Williams (dir.), Postcolonial approaches to the European Middle Ages: translating cultures, Cambridge, Cambridge University Press, 2005, p. 25-47.

4. Dans la suite comme ici, les mots Bretons et Bretagne renvoient à la province romaine historique (43-410 ap. J.-C.), soit l'ensemble de l'île aujourd'hui nommée Grande-Bretagne actuelle excepté le nord de l'Écosse. (N.d.T.)

5. Homi K. Bhabha, «DissemiNation : temps, récit et les marges de la nation moderne », dans Les Lieux de la culture : une théorie postcoloniale (trad. de l'anglais par Françoise Bouillot), Paris, Payot, 2007 [1994], p. 252.

6. Voir Kathleen Biddick, The Shock of Medievalism, Durham, Newcastle et Londres, Durham University Press, 1998; Jeffery Jerome Cohen (dir.), The Postcolonial Middle Ages, New York, St Martins Press, 2000 ; Carolyn Dinshaw, Getting Medieval: Sexualites Pre and Post-Modern, Durham, Newcastle et Londres, Duke University Press, 1999 ; Kathleen Davis, « National Writing in the Ninth Century : A Reminder for Postcolonial Thinking about the Nation », Journal of Medieval and Early Modern Studies, 28 (1998), p. 611-37 ; Michelle Warren et Patricia Ingham (dir.), Postcolonial Moves: Medieval through Modern, New York, Macmillan, 2003. 


\section{Labyrinthe, $n^{\circ} 35$}

7. Dans mon étude en cours : «Imperial Medievalism and the Formation of Modernity : Land, Language, Law » [titre de travail 2010, N.d.T.]. Voir aussi Thomas Metcalf, Ideologies of the Raj, The New Cambridge History of India, III.4, Cambridge, Cambridge University Press, 1995, p. 68-80.

8. Brian Young, " "The Lust of Empire and Religious Hate" : Christianity, History and India, 17901820 », dans Stefan Collini, Richard Whatmore, Brian Young (dir.), History, Religion and Culture : British Intellectual History 1750-1950, Cambridge, Cambridge University Press, 2000, p. 91. Sur la vie du capitaine Luard, voir James D. Lunt, Scarlet Lancer, Londres, R. Hart-Davis, 1964.

9. Voir plus bas, p. 104.

10. L'expression est d'Allen J. Frantzen, The Desire for Origins: New Language, Old English and Teaching the Tradition, New Brunswick / Londres, Rutgers University Press, 1990.

11. Voir Rosane Rocher, Orientalism, Poetry, and the Millennium: The Checkered Life of Nathaniel Brassey Halhed, 1751-1830, Delhi, Motilal Banarsidass, 1983 ; Bernard Cohn, « The Command of Language and the Language of Command », dans Ranajit Guha (dir.), Subaltern Studies IV: Writings on South Asian History and Society, Delhi, OUP, 1985, p. 276-339 (sur Gilchrist, voir p. 300-304) ; Katherine Prior, Lance Brennan and Robin Haines, « Bad Language : English, Persian and other Esoteric Tongues in the Dismissal of Sir Edward Colebrooke as Resident of Delhi in 1829 », Modern Asian Studies, 35 (2001), p. 75-112 ; Garland Cannon, Kevin R. Brine (dir.), Objects of Enquiry: The Life, Contributions and Influence of Sir William Jones, 2746- 1794, New York / Londres, New York University Press, 1995.

12. B. Cohn, « Command », art. cit., p. 282. Voir aussi Carol A. Breckenridge, Peter Van der Veer (dir.), Orientalism and the Postcolonial Predicament: Perspectives on South Asia, Philadelphie, University of Pensylvania Press, 1993.

13. J. B. Gilchrist, Dictionary English and Hindostanee, Calcutta, Stuart and Cooper, 1787, p. Xx.

14. Il s'agit de mouvements créés en Angleterre à l'époque des guerres civiles de l'époque cromwellienne (1642-1651), sous le signe d'un activisme puritain d'orientation communiste : les Levellers réclamaient l'instauration de droits constitutionnels élargis en matière politique et religieuse ; les Diggers prônaient une réforme de l'ordre social par la création de communautés égalitaires chrétiennes proscrivant l'appropriation privée des terres (N.d.T.).

15. Voir Christopher Hill, «The Norman Yoke », dans Puritanism and Revolution: Studies in Interpretation of the English Revolution of the Seventeenth Century, Londres, Secker and Hudson 1958, p. 50-122 ; Samuel Kliger, The Goths in England : A Study in Seventeenth and Eighteenth Century Thought, New York, Octagon Books, 1972 ; Bernard Bailyn, The Ideological Origins of the American Revolution, Cambridge, Massachussetts, Harvard University Press, 1971.

16. An Historical Essay on the English Constitution, Londres, Edward and Charles Dilly, 1771, p. 9 10 .

17. Tom Paine, Political and Miscellaneous Works, éd. R. Carlile, Londres, 1819, p. 16.

18. Histoire de la conquête de l'Angleterre par les Normands, dans Euvres d'Augustin Thierry, Bruxelles, Hauman et Cie, 1839 [1825], p. 361. Voir aussi Lionel Gossman, «Augustin Thierry and Liberal Historiography », dans Between History and Literature (Cambridge / Londres, Harvard University Press, 1990, p. 83-151).

19. Euvres d'Augustin Thierry, op. cit., p. 373.

20. Ibid., p. 7.

21. Franco Venturi, « Oriental Despotism », Journal of the History of Ideas, 24 (1963), p. 133-142, et Sylvia Tomaselli, « The Enlightenment Debate on Women », History Workshop, 20 (1985), p. 101124.

22. Voir Norbert Peabody, «Tod's Rajast'han and the Boundaries of Imperial Rule in $18^{\text {th }}$ Century India », Modern Asian Studies, 30 (1986), p. 185-220.

23. Voir la position sensiblement différente exprimée par T. Metcalf, Ideologies, op. cit., p. 14 : « Le projet orientaliste tel qu'il se développa répondait néanmoins clairement aux besoins de l'Europe. Toute forme de classification charrie avec elle un présupposé hiérarchique ». 


\section{Translations analogiques}

24. Sur le procès d'Hastings [premier gouverneur-général de l'Inde britannique (1773-1785), accusé de corruption et autres malversations à son retour, mais finalement acquitté par le Chambre des Communes, N.d.T.], voir Sara Suleri, The Rhetoric of English India, Chicago, Chicago University Press, p. 49-74. Ce moment historique est plaisamment raconté par William Dalrymple dans White Mughals : Love and Betrayal in Eighteenth-Century India, Londres, Flamingo, 2003.

25. Voir Eric Stokes, The English Utilitarians and India, Oxford, Oxford University Press, 1959 ; Uday Singh Mehta, Liberalism and Empire: A Study in Nineteenth-Century British Liberal Thought, Chicago / Londres, Chicago University Press, 1999.

26. Comme l'a récemment analysé Gauri Vishwanathan, Masks of Conquest : Literary Study and British Rule in India, Oxford, Oxford University Press, 1989.

27. Thomas Babington Macaulay, Selected Writings (éd. John Clive et Thomas Pinney), Chicago / Londres, Chicago University Press, 1972, p. 43.

278 Alexander Duff, Missionary Addresses, 1835-1839, Édimbourg, Johnstone and Hunt, 1850, p. 43.

29. B. Cohn, «Command », art. cit., p. 296.

30. C. E. Trevelyan, Treatise on the Education of the People of India, Londres, Longman et al., 1838, p. 23.

31. A. Duff, Missionary Addresses, op. cit., p. 15.

32. « Of Government Education in Bengal », dans Calcutta Review, V, III (1845), p. 221.

33. Parliamentary Papers, novembre 1852- août 1853, t. 32, Appendice N, p. 177.

34. Vortigern était un seigneur romano-britannique du v ve siècle ap. J.-C., réputé s'être appuyé sur des troupes de mercenaires anglo-saxons, les incitant à s'établir en Bretagne après le départ des Romains. (N.d.T.)

35. Linda Colley, Britons : Forging the Nation, 1707-1837, New Haven / Londres, Yale University Press, 1992 ; Leigh Davis, Acts of Union. Scotland and the Literary Negotiation of the British Nation, 1707-1803, Stanford, Stanford University Press, 1999.

36. John Luard, A History of the Dress of the British Soldier: From the Earliest Period to the Present Time, Londres, William Clowes and Sons, 1852 (rééd. Londres, Muller, 1971).

37. Isaac Disraeli, Amenities of Literature, Londres, Edward Moxson, 3 vol., t. I, 1841, p. 27.

38. A. Duff, Missionary Addresses, op. cit., p. 78-79.

39. Edward Augustus Freeman, Comparative Politics : six lectures read before the Royal Institution in January and February 1873, Londres, Macmillan, 1873, p. 45-47.

40. E. A. Freeman, Historical Essays, Londres, Macmillan, 1871, p. 51.

41. E. A. Freeman, The History of the Norman Conquest and its Results, Oxford, Clarendon, 6 vol., 1867-79, I, p. 604.

42. John Mitchell Kemble, The Saxons in England, Londres, Longman et al., 1849, p. 6.

43. E. A. Freeman, History of the Norman Conquest, op. cit., p. 20-21.

44. Voir Reginald Horsman, Race and Manifest Destiny, Cambridge / Londres, Harvard University Press, 1981.

45. Voir, à son propos, Hans Aarsleff, The Study of Language in England, 1780-1860, Minneapolis / Londres, University of Minnesota Press / Athlone, 1983, p. 193-1944 et 201-202, ainsi que John Mitchell Kemble and Jakob Grimm: A Correspondence 1832-1852 (éd et trad. Raymond A. Wiley), Leiden, Brill, 1971.

46. C. E. Trevelyan, Treatise, op. cit., p. 39.

47. E. A. Freeman, Comparative Politics, op. cit., p. 44.

48. Ibid., p. 38. Concernant l'indo-européanisme, l'aryanisme et l'empire, voir Sheldon Pollock, « Deep Orientalism : Notes on Sanskrit and Power beyond the Raj », dans Breckenridge et Van der Veer (dir.), Orientalism, op. cit., p. 45-75 ; Thomas R. Trautmann, Aryans and British India, Berkeley, University of California Press, 1997 ; Tony Ballantyne, Orientalism and Race : Aryanism in the British Empire, Basingstoke, Palgrave, 2001.

49. E. A. Freeman, Comparative Politics, op. cit., p. 43. 


\section{Labyrinthe, $n^{\circ} 35$}

50. Ibid., p. 47-48.

51. Ibid., p. 48.

52. Ibid.

53. Ibid., p. 44.

54. Ibid., p. 50.

55. Reginald Heber, « Morte D' Arthur », Canto III, I, The Poetical Works of Reginald Heber (Londres, John Murray, 1841), p. 249. Concernant Arthur en contexte impérial, voir Stephanie Barczewski, Myth and National Identity in Nineteenth Century Britain: The Legends of King Arthur and Robin Hood, Oxford, Oxford University Press, 2000, p. 201-230. 